\title{
Statistical mechanics in the extended Gaussian ensemble
}

\author{
Ramandeep S. Johal, ${ }^{*}$ Antoni Planes ${ }^{\dagger}$ and Eduard Vives ${ }^{\ddagger}$ \\ Departament d'Estructura i Constituents de la Matèria, Facultat de Física, Universitat de Barcelona, Diagonal 647, \\ 08028 Barcelona, Catalonia, Spain
}

(Received 5 August 2003; published 20 November 2003)

\begin{abstract}
The extended Gaussian ensemble (EGE) is introduced as a generalization of the canonical ensemble. This ensemble is a further extension of the Gaussian ensemble introduced by Hetherington [J. Low Temp. Phys. 66, 145 (1987)]. The statistical mechanical formalism is derived both from the analysis of the system attached to a finite reservoir and from the maximum statistical entropy principle. The probability of each microstate depends on two parameters $\beta$ and $\gamma$ which allow one to fix, independently, the mean energy of the system and the energy fluctuations, respectively. We establish the Legendre transform structure for the generalized thermodynamic potential and propose a stability criterion. We also compare the EGE probability distribution with the $q$-exponential distribution. As an example, an application to a system with few independent spins is presented.
\end{abstract}

DOI: 10.1103/PhysRevE.68.056113

PACS number(s): 05.70.-a, 05.20.Gg

\section{INTRODUCTION}

The development of statistical mechanics based on ensemble theory is founded on the postulate of "equal a priori probabilities," which is assumed to apply to all microstates consistent with the given macrostate of an isolated system [1]. The corresponding statistical ensemble is the so-called microcanonical ensemble. A representative system in this ensemble has all "mechanical" variables such as energy $E$, volume $V$, magnetization $M$, etc., fixed. For convenience in calculations, other ensembles are used which invariably suppose the existence of a subsidiary system or reservoir in contact with the actual system. For instance, in the canonical ensemble the walls of the system permit an exchange of energy with the reservoir while in the grand canonical ensemble, both energy and matter can be exchanged. In general, the different ensembles are constructed by allowing one or more mechanical variables to fluctuate. The exchange of each of these variables is controlled by a parameter which is a characteristic of the reservoir. For instance, in the case of the canonical ensemble, this parameter is precisely the temperature of the reservoir and determines the mean energy of the system. Actually, this is adequate when the reservoir is a very large system that can exchange arbitrary amounts of energy, without modification of its intensive properties. In practical situations, this is not always the case. However, very few studies have been devoted to analyze the consequences of possible deviations from these ideal reservoir properties.

In this paper, we develop the statistical mechanics of a system that can exchange energy with a finite reservoir characterized by two parameters: $\beta$ and $\gamma$. These parameters control independently the mean energy of the system and its energy fluctuations, respectively. The corresponding statistical ensemble represents a generalization of the canonical en-

\footnotetext{
*Electronic address: rjohal@ecm.ub.es

†Electronic address: toni@ecm.ub.es

‡Electronic address: eduard@ecm.ub.es
}

semble and will be called the extended Gaussian ensemble (EGE). A similar ensemble was already developed, in a more restricted framework, by Hetherington [2]. The author considered that the sample system was in contact with a finite reservoir with size dependent properties. The so-called Gaussian ensemble was introduced so that it is equivalent to the canonical ensemble in the limit of large systems, except in the energy range of a first-order transition. Interestingly, it enables a smooth interpolation between the microcanonical and the canonical ensembles. Taking into account these features, Challa and Hetherington $[3,4]$ showed the interest of this ensemble for Monte Carlo simulation studies of phase transitions. They demonstrated a significant reduction in computer time (compared to standard simulations in the canonical ensemble) and its adequacy for distinguishing second-order from first-order transitions. Compared to the EGE introduced in the present paper, the main difference arises from the fact that in the Gaussian ensemble the sample and the reservoir are assumed to be statistically independent which implies the additivity of the corresponding entropies. This is not assumed in our formalism. The consequences are important and will be discussed in depth in this work.

The present formalism can be considered as an alternative to the statistical mechanics based on nonadditive generalized entropies. Actually the study of such generalized entropies has generated a lot of interest in the past 15 years. The motivation for the so-called Tsallis statistical mechanics has been to extend the standard Boltzmann-Gibbs framework to include nonextensive systems [5]. Among different interpretations, it has been suggested that Tsallis formalism corresponds to an ensemble describing a system attached to a finite reservoir [6,7]. Although a large number of papers have been published, the physical meaning of many related issues is still open to discussion [8,9]. The EGE formalism that we propose in this paper provides a clear and consistent framework for the statistical mechanics with a finite reservoir.

The paper is organized as follows: in Secs. II and III, the EGE is founded from the analysis of a contact with a finite reservoir and from the maximum statistical entropy principle, respectively. In Sec. IV, the main thermodynamic relations are derived. In Sec. V, we highlight the nonadditive nature of the thermodynamic formalism. In Sec. VI, a stabil- 
ity criterion is proposed. In Sec. VII, the equilibrium distributions of the EGE are compared with the $q$-exponential distributions. In Sec. VIII, we present an example of application to a system of independent spins. Finally, in Sec. IX we summarize and conclude.

\section{CONTACT WITH A FINITE RESERVOIR}

Let us consider a system (that we will call the sample) in contact with a reservoir. Let us call the energy of the sample $E_{1}$ and the energy of the reservoir $E_{2}$. The sample and the reservoir together form an isolated system so that $E=E_{1}$ $+E_{2}$ is constant. Let us also define $\Omega_{2}\left(E_{2}\right)$ as the number of microstates of the reservoir. Following Callen [10], the probability that the system 1 is in a certain microstate with energy $E_{1}$ is given by

$$
p_{1}\left(E_{1}\right)=\frac{\Omega_{2}\left(E-E_{1}\right)}{\Omega_{1+2}(E)},
$$

where $\Omega_{1+2}(E)$ is the total number of states available for the set $1+2$. (Note that we do not assume that $\Omega_{1+2}$ can be factorized as a product $\Omega_{1} \Omega_{2}$.) Let us define the entropy of the reservoir as

$$
S_{2}\left(E_{2}\right)=\ln \Omega_{2}\left(E_{2}\right) .
$$

(Throughout the paper we choose Boltzmann's constant $k_{B}$ $=1$, so that the entropy is dimensionless.) Therefore

$$
p_{1}\left(E_{1}\right)=\frac{e^{S_{2}\left(E-E_{1}\right)}}{\Omega_{1+2}(E)} .
$$

The energy of the sample will, in general, fluctuate. Let us call $U$ its mean (equilibrium) value. We can develop $S_{2}(E$ $-E_{1}$ ) around the equilibrium value $E-U$ as

$$
\begin{aligned}
S_{2}\left(E-E_{1}\right)= & S_{2}(E-U)+\left.\frac{d S_{2}}{d E_{2}}\right|_{E-U}\left(U-E_{1}\right) \\
& +\left.\frac{1}{2 !} \frac{d^{2} S_{2}}{d E_{2}^{2}}\right|_{E-U}\left(U-E_{1}\right)^{2}+O\left(U-E_{1}\right)^{3} .
\end{aligned}
$$

The derivatives in the right-hand side of this expression are quantities which depend only on the reservoir. We define

$$
\left.\frac{d S_{2}}{d E_{2}}\right|_{E-U}=\beta
$$

and

$$
\left.\frac{d^{2} S_{2}}{d E_{2}^{2}}\right|_{E-U}=-2 \gamma
$$

The standard canonical ensemble is characterized by an infinite reservoir with constant $\beta$ (independent of $E_{2}$ ), which implies $\gamma=0$ and there is no term beyond the first-order term in Eq. (4). However, in the present paper, we consider a more general finite reservoir for which $\gamma \neq 0$. Thus in this EGE, the reservoir is characterized by the pair of parameters $\beta$ and $\gamma$. The thermodynamic meaning of these parameters will be clarified in the following sections. To explicitly highlight the effects of this modification and also for the sake of simplicity, we assume that the cubic and higher-order terms vanish. Then substituting Eq. (4) in Eq. (3) and denoting the energies of the microstates of the sample by $\epsilon_{i}(i=1, \ldots, M)$, we obtain

$$
p_{i}=\frac{1}{Z_{G}} \exp \left[-\beta \epsilon_{i}-\gamma\left(\epsilon_{i}-U\right)^{2}\right],
$$

where the normalization constant $Z_{G}$ is given by

$$
Z_{G}=\sum_{i=1}^{M} \exp \left[-\beta \epsilon_{i}-\gamma\left(\epsilon_{i}-U\right)^{2}\right] .
$$

The subscript $G$ only indicates the "Gaussian" form of the probabilities. Note that $U$ is the mean energy and must be obtained self-consistently from the following equation:

$$
U Z_{G}=\sum_{i=1}^{M} \epsilon_{i} \exp \left[-\beta \epsilon_{i}-\gamma\left(\epsilon_{i}-U\right)^{2}\right]
$$

Equations (7), (8), and (9) reduce to the standard canonical ensemble definitions when $\gamma=0$. Therefore, it is natural to relate the parameter $\gamma$ with the finite size of the reservoir.

\section{MAXIMUM STATISTICAL ENTROPY PRINCIPLE}

In this section, we derive the probability law of Eq. (7) from different arguments. This leads to a better understanding of the parameters $\beta$ and $\gamma$ as parameters characterizing the "equilibrium" distribution of the sample. To derive the probability distribution from the maximum statistical entropy principle, we maximize the standard Gibbs-BoltzmannShannon entropy given by

$$
S_{G}=-\sum_{i=1}^{M} p_{i} \ln p_{i},
$$

subject to the constraints of normalization of the probability, the given mean value of the energy, and the fixed value of the fluctuations, respectively, as

$$
\sum_{i=1}^{M} p_{i}=1
$$

$$
\left\langle\epsilon_{i}\right\rangle \equiv \sum_{i=1}^{M} \epsilon_{i} p_{i}=U
$$

$$
\left\langle\left(\epsilon_{i}-U\right)^{2}\right\rangle \equiv \sum_{i=1}^{M}\left(\epsilon_{i}-U\right)^{2} p_{i}=W .
$$

Then the maximization procedure is done by introducing the Lagrange multipliers $\lambda, \beta$, and $\gamma$ for the respective constraints, and maximizing the following functional $\mathcal{L}$ : 


$$
\begin{aligned}
\mathcal{L}= & -\sum_{i} p_{i} \ln p_{i}-\lambda\left(\sum_{i} p_{i}-1\right)-\beta\left(\sum_{i} \epsilon_{i} p_{i}-U\right) \\
& -\gamma\left(\sum_{i}\left(\epsilon_{i}-U\right)^{2} p_{i}-W\right) .
\end{aligned}
$$

By requiring the condition

$$
\frac{\partial \mathcal{L}}{\partial p_{i}}=0,
$$

it is easy to see that the optimum form of the probability distribution is given by the expression in Eq. (7). Therefore $\beta$ and $\gamma$, within this context, are simply Lagrange multipliers that allow to fix, self-consistently, a mean value of the energy $U=\left\langle\epsilon_{i}\right\rangle$ and a specific value of the variance $W$ $=\left\langle\left(\epsilon_{i}-U\right)^{2}\right\rangle$.

\section{THERMODYNAMIC RELATIONS}

We define a (dimensionless) thermodynamic potential $\Phi(\beta, \gamma)$ as

$$
\Phi(\beta, \gamma)=\ln Z_{G} .
$$

By differentiating Eq. (8), it can be straightforwardly obtained that

$$
\begin{aligned}
& -\left(\frac{\partial \Phi}{\partial \beta}\right)_{\gamma}=U(\beta, \gamma), \\
& -\left(\frac{\partial \Phi}{\partial \gamma}\right)_{\beta}=W(\beta, \gamma) .
\end{aligned}
$$

The second derivative renders

$$
-\left(\frac{\partial^{2} \Phi}{\partial \beta^{2}}\right)_{\gamma}=-\left(\frac{\partial U}{\partial \beta}\right)_{\gamma}=\frac{1}{W^{-1}(\beta, \gamma)-2 \gamma},
$$

which represents a generalization of the standard formula for energy fluctuations in the canonical ensemble. It is natural to define the extended heat capacity as

$$
\mathcal{C} \equiv-\beta^{2}\left(\frac{\partial U}{\partial \beta}\right)_{\gamma}=\frac{\beta^{2} W}{1-2 \gamma W} .
$$

This equation is the same that was already derived in Ref. [4]. Note that, contrary to what happens in the standard canonical ensemble, the positivity of the fluctuations $W$ does not guarantee the positivity of $\mathcal{C}$.

For $\gamma \rightarrow 0$, it is seen that relations (17) and (19) go to the corresponding relations for the case of canonical ensemble. Also in this limit, from Eqs. (18) and (19) we get an interesting relation given by

$$
\lim _{\gamma \rightarrow 0}\left(\frac{\partial \Phi}{\partial \gamma}\right)_{\beta}=\left(\frac{\partial^{2} \Phi}{\partial \beta^{2}}\right)_{\gamma},
$$

which resembles in form a diffusion equation.

The entropy $S_{G}$ as given by Eq. (10) is the inverse Legendre transform of $\Phi(\beta, \gamma)$, and can be expressed as

$$
S_{G}(U, W)=\beta U+\gamma W+\Phi,
$$

whereby $S_{G}$ is a function of the specified values of the constraints, i.e., $U$ and $W$. Therefore we have the following thermodynamic relations:

$$
\begin{aligned}
& \left(\frac{\partial S_{G}}{\partial U}\right)_{W}=\beta, \\
& \left(\frac{\partial S_{G}}{\partial W}\right)_{U}=\gamma .
\end{aligned}
$$

\section{NONADDITIVITY}

We remark that although the thermodynamics of a system in the EGE is well defined by the equations in the preceding section, it is not straightforward to establish a mutual equilibrium condition for two different systems that would allow us to establish a zeroth law (or, equivalently, an intensive temperature) [11]. This problem is due to the nonadditive character of the potential $\Phi(\beta, \gamma)$. Let us consider two systems 1 and 2 with Hamiltonians $H_{1}$ and $H_{2}$. By applying the rules derived in the preceding sections independently to the two systems, one can derive the thermodynamic potentials $\Phi_{1}(\beta, \gamma)$ and $\Phi_{2}(\beta, \gamma)$ as well as the mean energies $U_{1}(\beta, \gamma)$ and $U_{2}(\beta, \gamma)$. One can then try to solve the composite system $1+2$ with Hamiltonian $H_{1}+H_{2}$. It is easy to verify that the new potential $\Phi_{1+2}(\beta, \gamma)$ satisfies

$$
\begin{aligned}
\Phi_{1+2}(\beta, \gamma)= & \Phi_{1}(\beta, \gamma)+\Phi_{2}(\beta, \gamma) \\
& -\ln \left\langle e^{\gamma\left[\left(H_{1}+H_{2}-U_{1+2}\right)^{2}-\left(H_{1}-U_{1}\right)^{2}-\left(H_{2}-U_{2}\right)^{2}\right]}\right\rangle,
\end{aligned}
$$

where $U_{1+2}$ is the mean energy of the composite system. Note that $U_{1+2}$ as well as the average indicated by the angular brackets are computed with the probability distribution corresponding to the composite system $1+2$ which, in general, cannot be written as a product of probability distributions for systems 1 and 2.

The average values are, in general, nonadditive $\left(U_{1+2}\right.$ $\left.\neq U_{1}+U_{2}\right)$. But even if additivity of $U$ is imposed, the potential $\Phi$ remains nonadditive. The correction term depends on the microscopic details of the two Hamiltonians $H_{1}$ and $\mathrm{H}_{2}$.

This lack of additivity does not allow us to define an equivalence relation of "mutual" equilibrium. Consider that two (noninteracting) systems 1 and 2 are, independently, in equilibrium with a bath characterized by parameters $\beta$ and $\gamma$. One cannot ensure that the composite system $1+2$ is in equilibrium with the bath. 


\section{STABILITY CRITERION}

In standard thermodynamics, the stability criterion $-\partial U / \partial \beta>0$ is derived from the condition of maximum entropy. The derivation [10] considers a partition of an isolated system into any two subsystems. By allowing the two subsystems to alter their energies at fixed total energy, one can analyze the entropy change when the system is (virtually) displaced out of equilibrium. The condition of the maximum total entropy allows to deduce that the equilibrium state corresponds to a state with homogeneous $\beta$ (equilibrium condition) and with $-\partial U / \partial \beta>0$ (stability condition). For the derivation, nevertheless, additivity of the entropy of the two subsystems must be used.

Within our new formalism, an additivity assumption cannot be used. Therefore it is not straightforward to establish a stability criterion. Although we cannot give a rigorous proof, in this section we provide some evidences that the same criterion $(-\partial U / \partial \beta>0)$ must hold.

First of all, it is interesting to remark that the requirement of Eq. (9) that allows us to find $U$ can be rewritten as an extremal condition. Consider the definition of an (dimensionless) "out-of-equilibrium" potential:

$$
\Psi(\beta, \gamma, U)=\ln Z
$$

Note that here we are considering $\beta, \gamma$, and $U$ as independent variables. $U$ shall not be regarded as the internal energy but as a parameter that allows virtual displacements out of equilibrium. It can be checked that the self-consistent equation (9) can then be written as

$$
\left(\frac{\partial \Psi}{\partial U}\right)_{\beta, \gamma}=0
$$

Therefore, only the extrema of $\Psi$ correspond to equilibrium solutions: $U=\widetilde{U}(\beta, \gamma)$. By substituting in $\Psi$ one gets the equilibrium potential:

$$
\Phi(\beta, \gamma)=\Psi(\beta, \gamma, \widetilde{U}(\beta, \gamma))
$$

Second, from Eq. (8) note that if $\gamma>0$ and $U \rightarrow \pm \infty$, then $Z_{G} \rightarrow 0$ and therefore $\Psi \rightarrow-\infty$.

We can use this out-of-equilibrium potential to define a stability criterion. It is straightforward to compute its second derivative:

$$
\left(\frac{\partial^{2} \Psi}{\partial U^{2}}\right)_{\beta, \gamma}=2 \gamma(2 \gamma W-1) .
$$

Note that the positivity of $\mathcal{C}$ in Eq. (20) would guarantee that this second derivative of $\Psi$ is negative and therefore the state of equilibrium corresponds to maxima of $\Psi(\beta, \gamma, U)$ with respect to $U$ displacements. Although, contrary to what happens in the standard canonical ensemble, the positivity of $W$ does not ensure the sign of $-\partial U / \partial \beta$ in general, at least we can derive that for small and positive values of $\gamma$

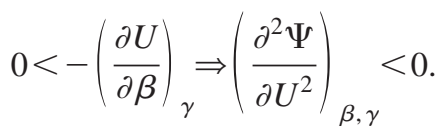

Thus we suggest that this is the stability criterion to be used within the EGE, and we will use it in Sec. VIII for the analysis of some examples.

\section{COMPARISON WITH $q$-EXPONENTIAL DISTRIBUTIONS}

$q$-exponential distributions are the central predictions of the generalized statistical mechanics proposed by Tsallis [5]. These distributions have been considered as model distributions to describe various complex systems at their stationary states [12-14]. The general form of such distributions is given by $p(x) \sim e_{q}(x)$, where the $q$ exponential is defined as $e_{q}(x)=[1+(1-q) x]^{1 /(1-q)}$. This function goes to the usual $\exp (x)$ function for $q \rightarrow 1$. For definiteness, we restrict to the range $0<q<1$.

In this section, we compare the $q$-exponential distributions with the equilibrium distributions of the EGE. But first, we show how to derive the $q$-exponential distributions by generalizing the canonical ensemble approach, along the lines of Sec. II. We define a parameter which is, in general, a function of the energy $E_{2}$ of the reservoir

$$
\beta\left(E_{2}\right)=\frac{d S_{2}}{d E_{2}} .
$$

At equilibrium, it attains the value given by Eq. (5). We impose that $\beta\left(E_{2}\right)$ satisfies

$$
\frac{d}{d E_{2}}\left(\frac{1}{\beta\left(E_{2}\right)}\right)=Q,
$$

where $Q$ is a positive valued constant. From Eqs. (32) and (31), we obtain

$$
\frac{d}{d E_{2}} \beta\left(E_{2}\right)=\frac{d^{2} S_{2}}{d E_{2}^{2}}=-Q \beta^{2}\left(E_{2}\right) .
$$

In general, for all integer values of $n$

$$
\frac{d^{n} S_{2}}{d E_{2}^{n}}=(n-1) !(-Q)^{n-1} \beta^{n}\left(E_{2}\right)
$$

Now unlike in Eq. (4), if in the expansion of $S\left(E-E_{1}\right)$ around the equilibrium value $(E-U)$ we retain derivatives of $S_{2}$ up to all orders, then we have

$$
S_{2}\left(E-E_{1}\right)=S_{2}(E-U)+\left.\sum_{n=1}^{\infty} \frac{1}{n !} \frac{d^{n} S_{2}}{d E_{2}^{n}}\right|_{E-U}\left(U-E_{1}\right)^{n} .
$$

On applying Eq. (34) for the case of equilibrium, we can write 


$$
S_{2}\left(E-E_{1}\right)=S_{2}(E-U)+\sum_{n=1}^{\infty} \frac{1}{n}(-Q)^{n-1} \beta^{n}\left(U-E_{1}\right)^{n},
$$

where note that $\beta$ is given by its value at equilibrium. The equilibrium probability distribution is then given from (3) as

$$
p\left(E_{1}\right) \sim \exp \left[\sum_{n=1}^{\infty} \frac{1}{n}(-Q)^{n-1} \beta^{n}\left(U-E_{1}\right)^{n}\right] .
$$

To compare Eq. (37) with the $q$-exponential distribution given by

$$
p_{q}\left(E_{1}\right) \sim e_{q}\left[\beta\left(U-E_{1}\right)\right]=\left[1+(1-q) \beta\left(U-E_{1}\right)\right]^{1 /(1-q)},
$$

we rewrite the $q$ exponential as

$$
e_{q}\left[\beta\left(U-E_{1}\right)\right]=\exp \left[\frac{\ln \left[1+(1-q) \beta\left(U-E_{1}\right)\right]}{(1-q)}\right],
$$

and expand the $\ln$ function using the series $\ln [1+x]=x$ $-x^{2} / 2+x^{3} / 3-x^{4} / 4+\cdots$, provided that $-1<x \leqslant 1$. Thus we can write

$$
e_{q}\left[\beta\left(U-E_{1}\right)\right]=\exp \left[\sum_{n=1}^{\infty} \frac{1}{n}\{-(1-q)\}^{n-1} \beta^{n}\left(U-E_{1}\right)^{n}\right],
$$

for $-1<(1-q) \beta\left(U-E_{1}\right) \leqslant 1$. Thereby, on identifying $Q$ $=(1-q)$ we may say that the general equilibrium distribution of Eq. (37) based on assumptions (31) and (32), is identical to a $q$-exponential distribution. Assuming that the relevant $q$ values are quite close to unity, we may keep terms only up to second order as done in Eq. (4). Then the equilibrium $q$ distribution for system 1 being in microstate $i$ of energy $\epsilon_{i}$ can be written as

$$
p_{q}\left(\epsilon_{i}\right)=\frac{1}{Z_{q}} \exp \left[-\beta \epsilon_{i}-\frac{1}{2}(1-q) \beta^{2}\left(\epsilon_{i}-U\right)^{2}\right],
$$

where $Z_{q}$ is the normalization constant.

On the other hand, for the case of EGE, instead of fixing the derivative of $\beta^{-1}$ [Eq. (32)], we fix the derivative of $\beta$ as follows:

$$
\frac{d}{d E_{2}} \beta\left(E_{2}\right)=-2 \gamma
$$

where $\gamma$ is independent of $E_{2}$. This ensures that the higherorder $(n>2)$ derivatives of $S_{2}$ vanish. On comparing Eqs. (41) and (7), we note that $(1-q)$ plays the role analogous to $\gamma$.

It may be remarked that if we identify parameter $\beta\left(E_{2}\right)$ $=1 / T\left(E_{2}\right)$ as the inverse temperature, then Eq. (32) implies that the heat capacity of the reservoir $C_{2}=d E_{2} / d T=Q^{-1}$. Recently, the $q$-exponential distributions have been discussed in the context of a reservoir with finite heat capacity [6]. On the other hand, following Gibbs' approach to the canonical

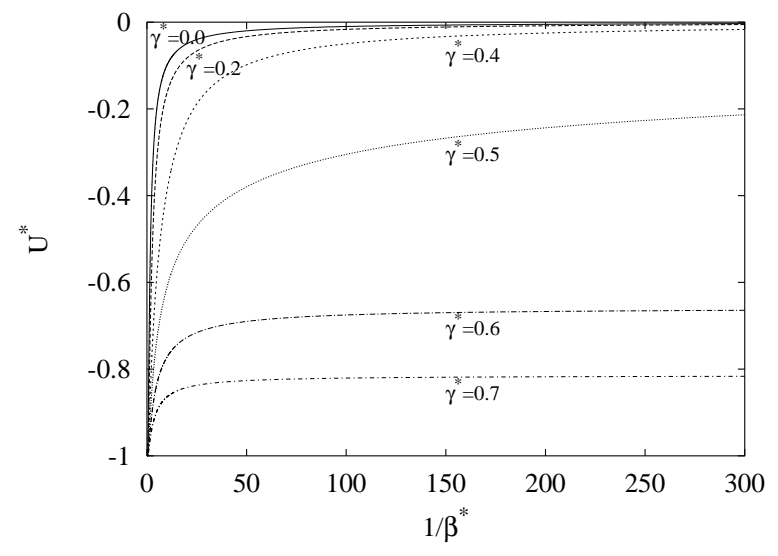

FIG. 1. Behavior of the mean reduced energy $U^{*}$ as a function of $1 / \beta^{*}$ for several values of $\gamma^{*}$ in a system of a single spin.

ensemble, but instead using the $q$-generalized Boltzmann entropy, $q$-exponential distributions were derived in Ref. [15].

\section{APPLICATION TO A SYSTEM OF INDEPENDENT SPINS}

\section{A. Single spin}

As a first example of the EGE, we apply our formalism to the problem of a system with only two energy levels. Let us consider a single spin $s= \pm 1$ in the presence of a constant external magnetic field $B$. The Hamiltonian of the system reads

$$
H=-B s .
$$

The partition function is given by

$$
Z_{G}=e^{\beta B} e^{-\gamma(-B-U)^{2}}+e^{-\beta B} e^{-\gamma(B-U)^{2}},
$$

where the mean energy $U$ is the solution of the following self-consistent equation:

$$
U=-B e^{\beta B} e^{-\gamma(-B-U)^{2}}+B e^{-\beta B} e^{-\gamma(B-U)^{2}} .
$$

The dependence on $B$ can be easily overcome by defining the reduced units (dimensionless quantities):

$$
U^{*}=U / B, \quad \beta^{*}=\beta B, \quad \gamma^{*}=\gamma B^{2} .
$$

Thus, Eq. (45) becomes

$$
U^{*}=e^{-\beta^{*}} e^{-\gamma^{*}\left(1-U^{*}\right)^{2}}-e^{\beta^{*}} e^{-\gamma^{*}\left(1+U^{*}\right)^{2}} .
$$

The numerical solution of this equation is plotted in Fig. 1 . The behavior of $U^{*}$ as a function of $1 / \beta^{*}$ is shown for different values of $\gamma^{*}$. For $\gamma^{*}=0$, one recovers the behavior $U^{*}=\tanh \left(\beta^{*}\right)$ corresponding to the case of a system in contact with an infinite reservoir. For $\gamma^{*} \neq 0, U^{*}$ is smaller, indicating that it is more difficult to disorder the system by decreasing $\beta^{*}$. It is interesting to note that for $\gamma^{*} \simeq 0.5$, there is a change in the behavior at $\beta^{*} \rightarrow 0$. Above this value of $\gamma^{*}$, the system is not able to disorder completely anymore and always keeps a certain magnetization $(m=\langle s\rangle$ 


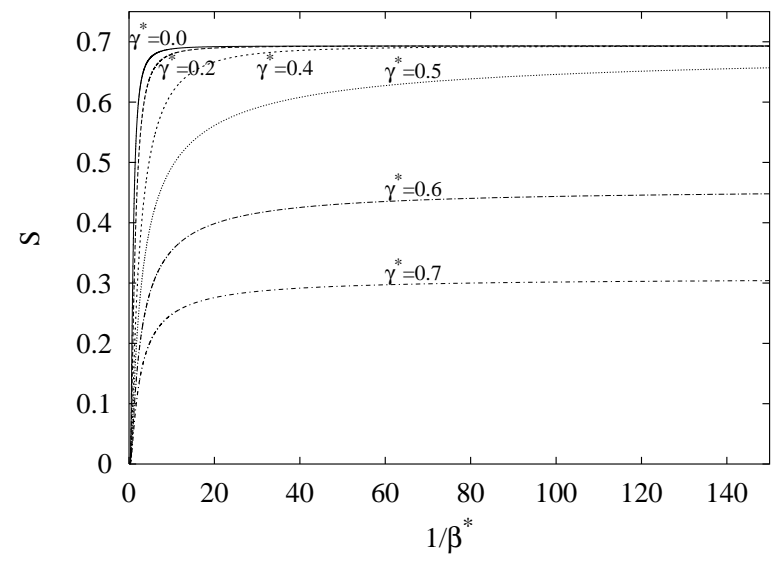

FIG. 2. Behavior of the entropy $S$ as a function of $1 / \beta^{*}$ for several values of $\gamma^{*}$ in a system of a single spin.

$\left.=-U^{*}\right)$. This can be regarded as a "phase transition" that occurs at $\beta^{*}=0$. This change in the behavior occuring at $\gamma^{*} \simeq 0.5$ can also be seen by plotting the entropy $S$ as a function of $1 / \beta^{*}$ for different values of $\gamma^{*}$. This is shown in Fig. 2. For $\gamma^{*}<0.5$ the entropy tends to $\ln 2$ for $\beta^{*} \rightarrow 0$, whereas it tends to a lower value for $\gamma^{*}>0.5$.

\section{B. Two spins}

As a second step, it is also very instructive to study a system of two independent spins. This will illustrate the nonextensive behavior of the solution. In this case, the numerical solution of the self-consistent equation (9) for the mean energy renders the behavior shown in Fig. 3. For the values of $\beta^{*}$ and $\gamma^{*}$ for which more than one solutions are possible, we have used the stability criterion proposed in Sec. VI to decide which is the "equilibrium" solution. As can be seen, for $\gamma^{*}>0.49$ a discontinuity occurs associated with a sudden loss of order in the system. Although the system is far from the thermodynamic limit, this change shares many similarities with a phase transition. Figure 4 displays the behavior of the corresponding energy fluctuations. It can be seen that $W^{*}=\left(\left\langle H^{2}\right\rangle-U^{2}\right) / B^{2}$ exhibits a cusp at the transition for

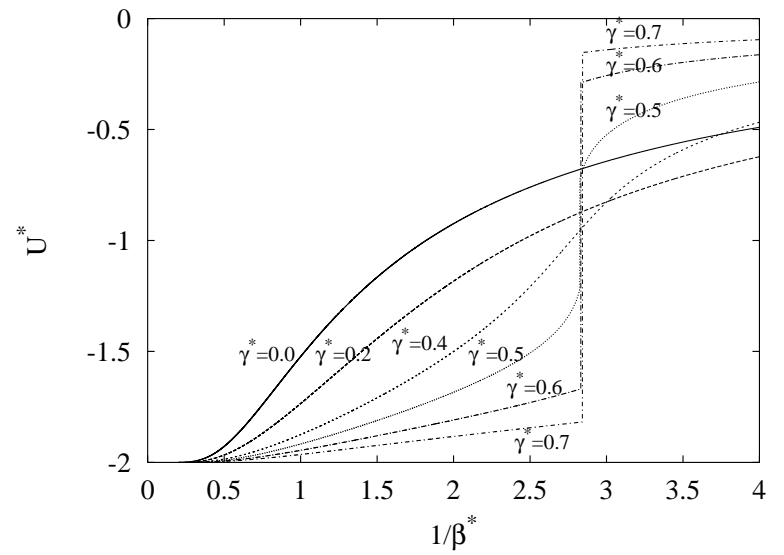

FIG. 3. Behavior of the mean energy $U^{*}$ as a function of $1 / \beta^{*}$ for several values of $\gamma^{*}$ in a system of two independent spins.

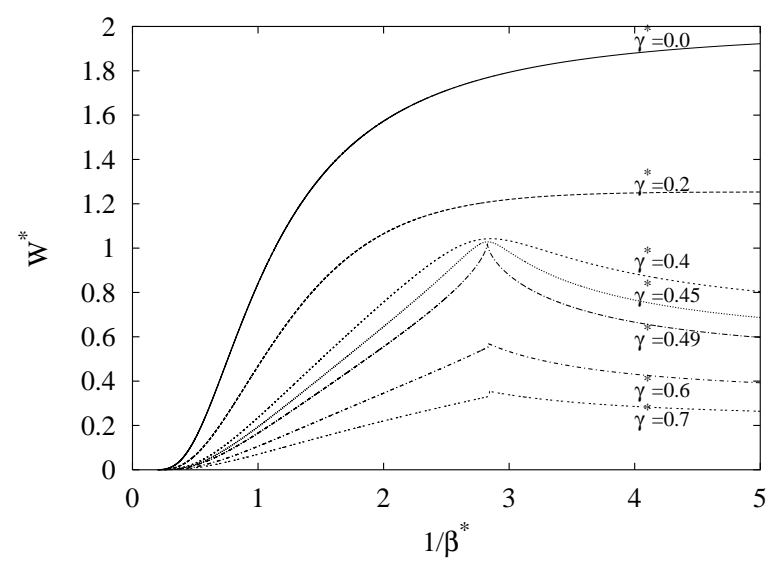

FIG. 4. Behavior of the energy fluctuations as a function of $1 / \beta^{*}$ for several values of $\gamma^{*}$ in a system of two independent spins.

$\gamma^{*} \simeq 0.49$. For larger values of $\gamma^{*}$, the fluctuations exhibit a discontinuity. The discontinuities are associated with firstorder phase transitions that display metastable behavior. As an example, in Fig. 5, we show the detailed behavior of $U^{*}$ as a function of $1 / \beta^{*}$ for $\gamma^{*}=0.6$. In the range $2.42<1 / \beta^{*}<2.86$, the numerical analysis of the selfconsistent equation renders three solutions.

By analyzing the behavior of the potential $\Psi\left(\beta^{*}, \gamma^{*}, U^{*}\right)$, shown in Fig. 6, it is easy to verify that two of such solutions are stable (correspond to local maxima of $\Psi$ ) whereas one is unstable (corresponds to a local minimum of $\Psi$ and is not plotted in Fig. 5). The equilibrium transition jump at $1 / \beta^{*} \simeq 2.832$ is determined by the equality of the two maxima of $\Psi$.

For the system of two spins, therefore, we can plot a $\beta^{*}-\gamma^{*}$ phase diagram, shown in Fig. 7. The line of first-order phase transitions ends in a "critical" point at $\beta^{*} \simeq 0.353$ and $\gamma^{*} \simeq 0.49$. This point is characterized by the condition $1 / W^{*}=2 \gamma^{*}$ and thus, according to Eq. (19), corresponds to a divergence of $\mathcal{C}$ but not to a divergence of the

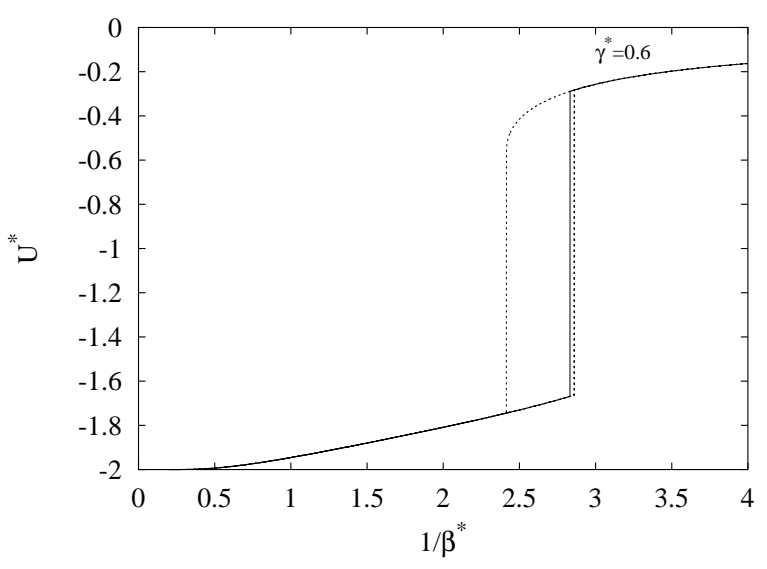

FIG. 5. Behavior of $U^{*}$ as a function of $1 / \beta^{*}$ for a system of two spins with $\gamma^{*}=0.6$. The continuous line represents the equilibrium solution (with maximum $\Psi$ ) and the dashed lines represent metastable solutions which correspond to local maxima. 


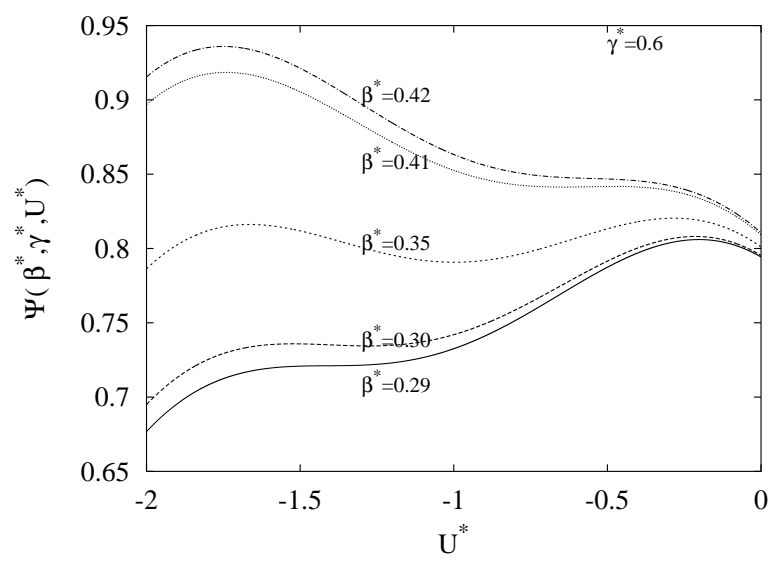

FIG. 6. Behavior of the potential $\Psi\left(\beta^{*}, \gamma^{*}, u^{*}\right)$ for different values of $1 / \beta^{*}$ for a system of two spins with $\gamma^{*}=0.6$. The equilibrium value of $U^{*}$ corresponds to the maximum of the potential $\Psi$.

fluctuations $W^{*}$, which can never diverge for such a system with a finite number of bounded energy levels.

\section{Several spins}

We have also performed a numerical study of systems with larger number of independent spins in the presence of an external field. An example is shown in Fig. 8, corresponding to a system with four spins (16 energy levels). A sequence of two consecutive phase transitions can be observed. As an interesting remark we want to note that in the case of $N$ "noninteracting" spins $s_{k}(k=1, \ldots, N)$ in the presence of an external field, long-range forces will appear due to the finite size of the bath. This can be easily seen by writing the probabilities $p_{i}$ for the microstates $\left(i=1, \ldots, 2^{N}\right)$ of such a system:

$$
p_{i}=\exp \left[\beta^{*} \sum_{k=1}^{N} s_{k}-\gamma^{*}\left(\sum_{k=1}^{N} s_{k}-U^{*}\right)^{2}\right] .
$$

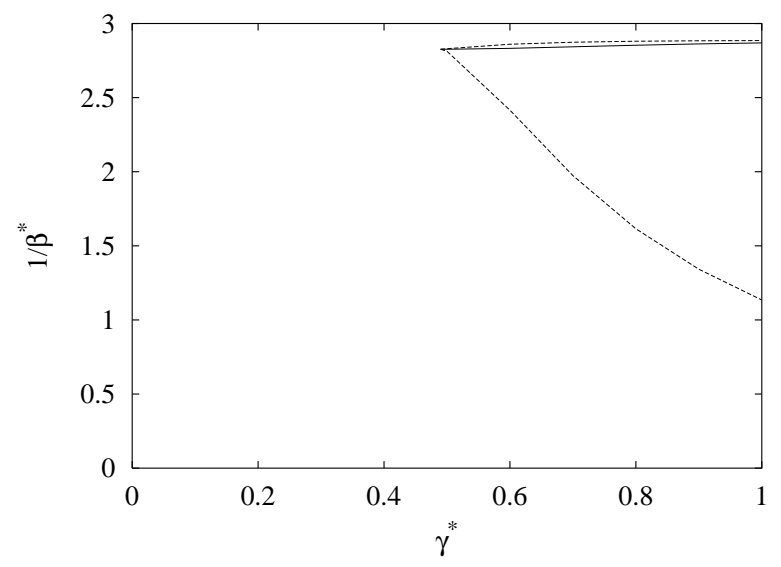

FIG. 7. Phase diagram for a system of two spins. The continuous line represents first-order transitions whereas discontinuous lines indicate the metastability limits.

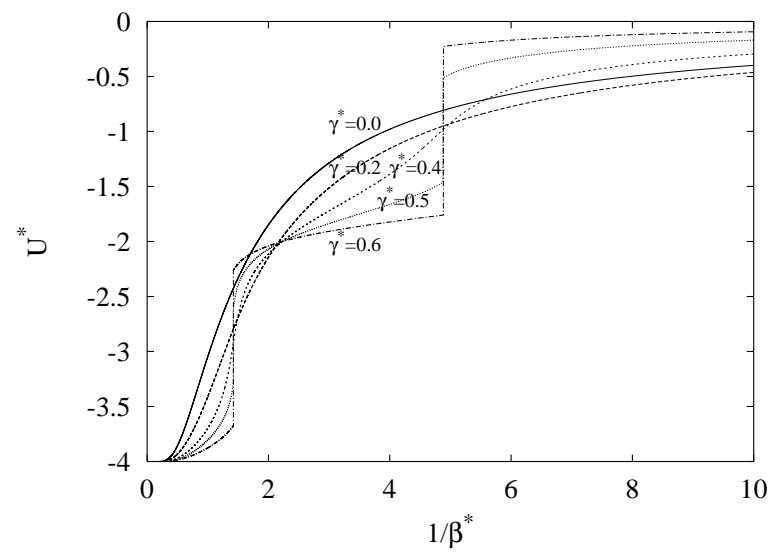

FIG. 8. Behavior of the mean energy $U^{*}$ as a function of $1 / \beta^{*}$ for several values of $\gamma^{*}$ in a system of four independent spins.

Note that the development of the squared term in the exponent will lead to terms $-\gamma^{*} s_{k} s_{j}$ which correspond to antiferromagnetic interactions among all spin pairs. A more detailed study of these examples is out of the scope of this paper.

\section{SUMMARY}

We have presented the EGE as a generalization of the standard canonical ensemble. The ensemble statistics have been derived by following two methods: first by considering a system in contact with a finite bath and second from the maximum statistical entropy principle by fixing the knowledge of both the mean energy and the energy fluctuations. The obtained probability law depends on two parameters $\beta$ and $\gamma$ which are properties of the bath. Thermodynamic relations have been derived and a possible stability criterion has been suggested. Nevertheless this point as well as the possibility for establishing a mutual equilibrium criterion will need further analysis in future works. We have also presented an application of the EGE formalism to the analysis of a system of one spin and two independent spins. Among other interesting results, the most remarkable one is the possibility for occurrence of a critical point or first-order phase transitions induced by the finite size of the reservoir. Further, comparisons of this new ensemble formalism with alternative extensions of statistical mechanics proposed for the study of nanosystems [16-18] or for other nonextensive systems are interesting problems for research.

\section{ACKNOWLEDGMENTS}

This work received financial support from CICyT (Spain), Project No. MAT2001-3251, and CIRIT (Catalonia), Project No. 2000SGR00025. R.S.J. acknowledges financial support from Secretaria de Estado de Educacion y Universidades (Spanish Ministry of Education, Culture and Sports). The authors thank Dr. Avadh Saxena for fruitful discussions and Dr. J. M. Sancho for a critical reading of the manuscript. 
[1] R.K. Pathria, Statistical Mechanics, 2nd ed. (Butterworth Heinemann, Oxford, 1996).

[2] J.H. Hetherington, J. Low Temp. Phys. 66, 145 (1987).

[3] M.S.S. Challa and J.H. Hetherington, Phys. Rev. Lett. 60, 77 (1988).

[4] M.S.S. Challa and J.H. Hetherington, Phys. Rev. A 38, 6324 (1988).

[5] C. Tsallis, J. Stat. Phys. 52, 479 (1988).

[6] M.P. Almeida, Physica A 300, 424 (2001).

[7] A.R. Plastino and A. Plastino, Phys. Lett. A 193, 140 (1994).

[8] M. Nauenberg, Phys. Rev. E 67, 036114 (2003).

[9] C. Tsallis, e-print cond-mat/0304696.

[10] H.B. Callen, Thermodynamics and an Introduction to Thermostatistics, 2nd ed. (Wiley, New York, 1985).

[11] W.J. Hornix, in A Critical Review of Thermodynamics, edited by E.B. Stuart, B. Gal-Or, and A.J. Brainard (Mono Book Cor- poration, Baltimore, 1970)

[12] Nonextensive Statistical Mechanics and Its Applications, edited by S. Abe and Y. Okamoto, Lecture Notes in Physics Vol. 560 (Springer-Verlag, Heidelberg, 2001).

[13] Non-Extensive Statistical Mechanics and Physical Applications, edited by G. Kaniadakis, M. Lissia, and A. Rapisarda [Physica A 305, 1 (2002)].

[14] Nonextensive Entropy-Interdisciplinary Applications, edited by M. Gell-Mann and C. Tsallis (Oxford University Press, Oxford, 2003).

[15] S. Abe and A.K. Rajagopal, Europhys. Lett. 55, 6 (2001).

[16] T.L. Hill, Thermodynamics of Small Systems (Dover, New York, 1994).

[17] T.L. Hill, Nano Lett. 1, 111 (2001).

[18] T.L. Hill, Nano Lett. 1, 273 (2001). 